\title{
THE FETAL HYPOTHALAMUS AND PITUITARY DURING GROWTH AND DIFFERENTIATION
}

\author{
D. F. Swaab*, F. W. van Leeuwen*, J. Dogterom* and W. J. Honnebier $\dagger$ \\ *Netherlands Central Institute for Brain Research, Ijdijk 28, Amsterdam, and $†$ Department of Obstetrics \\ and Gynaecology, University Clinic Wilhelmina Gasthuis, Amsterdam, The Netherlands
}

\section{SUMMARY}

In our project on the involvement of the fetal brain and pituitary in intrauterine growth and parturition, data on human anencephalics are compared with those obtained in experiments with rat fetuses.

An intrauterine growth spurt was observed in intact rat fetuses (starting at day 19 of pregnancy) and normal human fetuses (around 20 weeks of pregnancy), which appeared to be dependent on the integrity of the fetal brain and pituitary. Rat experiments showed that the acceleration of intrauterine growth was caused by endogenous fetal $\alpha$-melanocyte-stimulating hormone $(\alpha-\mathrm{MSH})$. This hormone is also shown to be present in normal human fetal pituitaries during the intrauterine growth spurt. This, together with the absence of $\alpha$-MSH fluorescence in anencephalic pituitaries suggests the involvement of $\alpha-\mathrm{MSH}$ also in human fetal growth.

The normal course of labour appeared also to be dependent on the integrity of the fetal brain and pituitary in man and rat. Since a protracted course of labour was found in Brattleboro rats being homozygous for diabetes insipidus, the fetal hypothalamo-neurohypophyseal system was thought to stimulate the course of labour. However, using immunofluorescence techniques, that enabled the specific localization of oxytocin and vasopressin in the adult rat, these hormones were not observed in the pituitary of fetal Wistar rats near term. Instead, a cross reacting compound which was probably arginine-vasotocin (AVT) was found throughout the hypothalamo-neurohypophyseal system which, by immuno-electronmicroscopy, appeared to be localized within the neurosecretory vesicles of the fetal neurohypophysis. The presence of AVT was confirmed by radioimmunoassay. AVT was absent or only present in very small quantities in the pituitary of homozygous Brattleboro fetuses. AVT from the fetus may play a role in the course of normal delivery. The presence of AVT in the fetal rat neurohypophysis and of $\alpha$-MSH in the intermediate lobe of the fetal human pituitary shows, that fetal endocrine systems might be different from those, operating in adult organisms.

\section{INTRODUCTION}

In the present paper two fetal peptide hormones are discussed: $\alpha$-melanocyte stimulating hormone $(\alpha-\mathrm{MSH})$ and its involvement in fetal growth and arginine-vasotocin (AVT) and its possible involvement in parturition.

Anencephalic children are studied in a project, meant to determine in which processes the human fetal brain and pituitary might be involved $[1,2]$. These data are compared to those in a rat model from which the fetal brain and pituitary were removed by aspiration [3]. This rat model enables us, in addition, to unravel the endocrine mechanisms involved in detail, while essential steps in these mechanisms are subsequently tested for their presence in human fetuses again.

Compared to the mechanisms involved in postnatal growth, very little is known about fetal factors that may influence intrauterine growth, although fetal growth disturbances are very important in view of their relation to perinatal mortality [4]. From various studies it appeared that fetal growth is regulated by mechanisms that are different from those operating in postnatal growth. For instance, growth hormone administered to rat fetuses resulted in a decrease of fetal body weight [5]. Somatomedin (kindly donated by Dr. Roger Guillemin, administered as $15 \mu \mathrm{g} / \mathrm{fetus}$ s.c. on day 19 of pregnancy) did not affect fetal body weight at all (Swaab and Honnebier, unpublished results). Moreover, it was not possible to increase fetal body weight by injection of a series of pituitary hormones or related compounds [6]. The only compound that could stimulate intrauterine growth in rat was $\alpha$-MSH (see below).

A second field of interest is the possible role of the fetal brain in the process of parturition. Data from human and rat showed that the fetal brain is involved in the course of labour rather than in its initiation. A hormone that might be involved in stimulating the course of labour is the fetal posterior lobe hormone arginine-vasotocin (AVT) [7].

The presence of AVT in the fetal rat hypothalamoneurohypophyseal system and of $\alpha$-MSH in the intermediate lobe of the human fetal pituitary are discussed in relation to endocrine differentiation.

\section{THE FETAL PITUITARY AND INTRAUTERINE GROWTH}

An acceleration of intrauterine growth rate was found around 20 weeks of pregnancy in man (Fig. 1, a) and at day 19 of pregnancy in rat (Fig. 1, c). This fetal "growth spurt" is dependent on the integrity of the fetal brain and pituitary (Fig. 1, b and d) as 


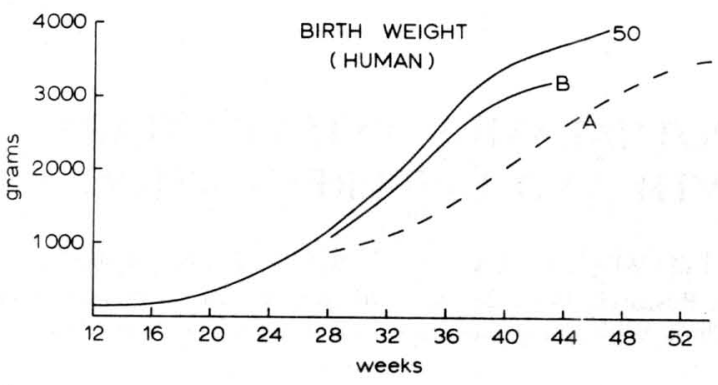

b.

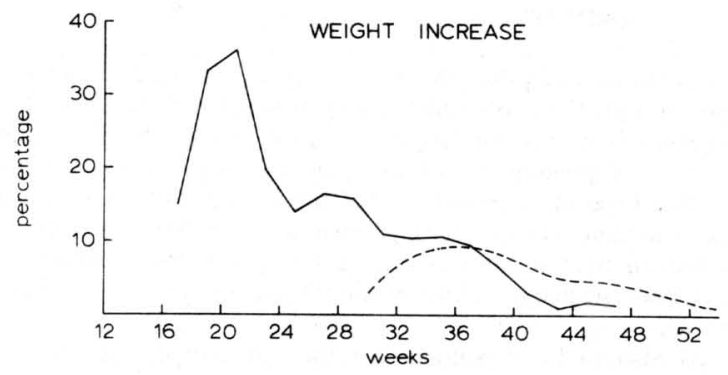

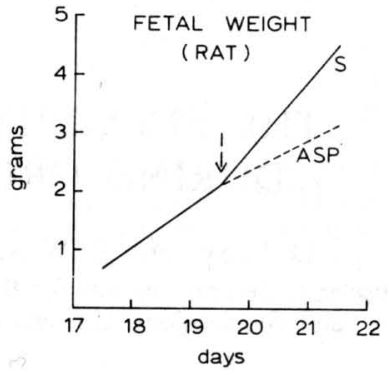

d.

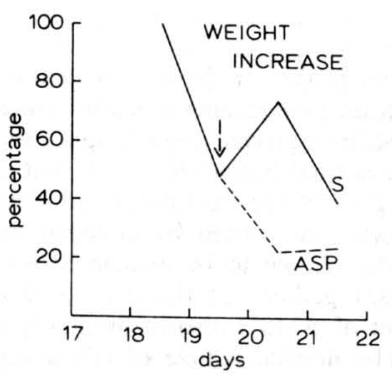

Fig. 1. The brain dependent intrauterine growth rate in man (left) and rat (right). (a) Birth weight (grams) by gestation length (weeks) in man. (1) Growth of normal human fetuses. The curve is the smoothed 50th percentile line (50) of Kloosterman's intrauterine growth curve $(20-47$ weeks of pregnancy, cf. [1]) which was extended with the medians of the birth weight of 91 children that were either born alive or died during birth between 12 and 19 weeks of pregnancy. (2) The 50 th percentile line of the control group after subtraction of the brain weight (B). (3) A computer calculated polynomial regression line of the birth weight of 122 anencephalics (broken line A) (cf. 1). Note the lower body weight of the anencephalics.

(b) Intrauterine growth rate given as percentage increase in body weight per week. (1) Normal intrauterine growth rate calculated from 15-47 weeks of pregnancy, using the 50th percentile data, and expressed as mean increase over a 2-week period. (2) Anencephalic growth rate based on line A and expressed as mean increase over a 2-week period. Note the intrauterine growth spurt around 20 weeks of pregnancy. Birth weights of anencephalics during this growth spurt are not present in our series. Clinical observations revealed, however, that growth retardation in anencephaly starts very early. The uterus was found to be too small for date for the first time in cases even at 13 weeks of pregnancy [6]. This together with the course of the anencephalic birth weight curve and growth rate curve make it probable that the normal intrauterine growth spurt is lacking in anencephalics.

(c) Intrauterine growth (grams) by gestation length (days) in rat. Body weight of fetuses that were sham operated (arrow) on day 19 (5, drawn line) and that of fetuses that were brain aspirated (ASP, broken line) on day 19 of pregnancy (data calculated from [3]). Note the increase in growth rate on day 19 in the sham operated group which is missing in the brain aspirated group.

(d) Growth rates, given as percentage increase in body weight per day, calculated from the data of (c). Note the intrauterine growth spurt in sham operated fetuses and its absence in brain aspirated fetuses.

appears from its absence in human anencephalics [1] and brain-aspirated rat fetuses [3]. A similar decrease in intrauterine growth rate has recently been described in rhesus monkey fetuses following removal of the fetal brain and pituitary [8]. The only compound that was able to stimulate fetal growth again in brain-aspirated rat fetuses was $\alpha$-MSH $[5,6]$. This hormone appeared in the intermediate lobe of the fetal pituitary one day before the start of the intrauterine growth spurt, as shown by both immunofluorescence microscopy and bioassay. In addition, a negative correlation was found between the pituitary content of MSH and fetal body weight on day 19 of pregnancy, suggesting an earlier onset of the release in the heavier fetuses. That endogenous fetal $\alpha$-MSH has indeed a function in stimulating intrauterine growth appeared from the growth inhibition follow- ing injection of purified anti- $\alpha$-MSH directly into the fetuses on the day of the intrauterine growth spurt [9].

As a first step to establish whether in man the same relationship exists between $\alpha$-MSH and intrauterine growth, we studied by means of immunofluorescence microscopy whether $\alpha$-MSH was present in the human fetal pituitary during the intrauterine growth spurt. Indeed, $\alpha-\mathrm{MSH}$ was found in the intermediate and anterior lobe of all 6 normal human fetuses that were studied between 15-20 weeks of pregnancy. In the two anencephalics examined until now this hormone appeared to be absent in the pituitary [10]. Since anencephalics show a lower intrauterine growth rate [1] and probably have lower MSH levels in blood [11], $\alpha$-MSH might play a role in intrauterine growth in man as well. (For a recent review see [4].) 
According to Silman et al. [12], $\alpha-\mathrm{MSH}$ is only present in the human fetal pituitary and not in that of adult man. This concurs with the results of other authors $[13,14]$ who found respectively no, or only minute, quantities of this hormone in pituitaries of adult man. Our finding of $\alpha$-MSH in the intermediate lobe of the pituitary of a human pregnant female [10] shows, however, that the disappearance of $\alpha$-MSH in adults is a reversible phenomenon. The absence of $\alpha$-MSH in the pituitary of human anencephalics and its presence in normal human fetuses [10] would point to the fetal brain as the stimulating structure for the appearance of this hormone.

\section{THE FETAL BRAIN AND PARTURITION}

In sheep the fetal pituitary can be considered as the trigger for the onset of labour [15]. In man and rat, however, the initiation of labour appeared to be less dependent on the fetal pituitary. In human anencephalic fetuses the mean gestation length was similar to that of the controls. Yet, since a high percentage of pre- and post-mature labours were found in anencephalics, the fetal brain may be involved in only the exact timing of the initiation of labour $[1,7]$. The same was found in rhesus monkeys in which experimental anencephaly did not influence the mean gestation length, yet the majority $(67 \%)$ of the monkeys delivered either pre- or post-term [16].

The course of labour was, however, found to be strongly dependent on the integrity of the fetal brain and pituitary in man and rat. A protracted course of labour was observed both in human anencephalics and in rat fetuses from which the brain and pituitary were removed. Since a similar disturbed course of labour was found in Brattleboro rats being homozygous for diabetes insipidus, the fetal neurohypophysis might play a role in the acceleration of the course of labour [7]. All the more since it was possible to evoke uterine contractions from the fetal side by injection of posterior lobe hormones $[2,17]$, and since extremely high levels of these hormones are present in umbilical cord blood [18]. A fluorescence technique that enabled specific immunolocalization of oxytocin and vasopressin in the hypothalamo-neurohypophyseal system (HNS) of the adult rat $[19,20]$ revealed that neither of these hormones could be localized in the Wistar fetal HNS at term. Instead, fluorescence that was observed using non-purified antibodies against vasopressin, was based on cross reaction with a closely related peptide. This cross reacting compound was also present in the pituitary of 1 -week-old chicks and human fetuses of about 20 weeks of pregnancy. This pointed to arginine-vasotocin (AVT) as the cross reacting compound, since this hormone, that differs only one amino acid from vasopressin and oxytocin is present in the pituitary of chick [21] and human fetuses [22,23]. A strong cross reaction of our anti-vasopressin plasmas with AVT was indeed found (Table 1) using the model system of synthetic hormones bound to agarose beads [19]. Following absorption of the antivasopressin plasma with agarose beads containing AVT, the fluorescence of the fetal Wistar pituitary had disappeared. Since

Table 1. Cross reactions of antivasopressin and antioxytocin with arginine-vasotocin (AVT)

\begin{tabular}{|c|c|c|c|}
\hline anti-AVP & on AVP beads & on AVT beads & \\
\hline 123 19/6/74 & $86(7.1)$ & $63(2.5)$ & \\
\hline $12419 / 12 / 73$ & $118(16.4)$ & $124(9.8)$ & \\
\hline $12524 / 12 / 72$ & $157(19.6)$ & $162(6.9)$ & \\
\hline $1268 / 6 / 2$ nd & $271(29.5)$ & $220(14.5)$ & \\
\hline $1277 / 5 / 73$ & $170(20.7)$ & 227 (11.7) & \\
\hline $758 \quad 28 / 5 / 74$ & $190(21.7)$ & $346(20.7)$ & \\
\hline anti-LVP & on AVP beads & on AVT beads & \\
\hline 118 19/3/74 & $48(5.0)$ & $69(3.8)$ & \\
\hline $11916 / 3 / 73$ & $102(11.0)$ & $95(9.0)$ & \\
\hline $12019 / 3$ & $131(7.0)$ & $96(4.3)$ & \\
\hline $12116 / 3 / 73$ & $76(6.1)$ & $96(5.9)$ & \\
\hline $1945 / 6 / 73$ & $48(3.3)$ & $47(2.9)$ & \\
\hline $757 \quad 10 / 3 / 73$ & $84(12.7)$ & $65(3.8)$ & \\
\hline anti-oxytocin & on AVP beads & on AVT beads & on oxytocin beads \\
\hline $\mathrm{O}-1 \mathrm{C}$ & $40(3.6)$ & $48(3.1)$ & $80(6.1)$ \\
\hline $\mathrm{O}-2 \mathrm{D}$ & $28(1.5)$ & $69(4.1)$ & $131(3.8)$ \\
\hline $\mathrm{O}-3 \mathrm{C}$ & $43(3.8)$ & $65(5.5)$ & $98(3.2)$ \\
\hline
\end{tabular}

The cross reaction of antiarginine-vasopressin (anti-AVP), antilysine-vasopressin (anti-LVP) and antioxytocin was measured by means of immunofluorescence microscopy on agarose beads containing synthetic AVP, AVT or oxytocin. All plasmas were diluted 1:80. The data are corrected for background readings obtained with 1:80 diluted control plasma. Note the strong cross reaction of all antibodies with AVT. 
Table 2. Neuropeptides in fetal pituitaries at term (in ng/pituitary \pm S.E.M.) of one litter $(n=10)$ of Wistar fetuses and one litter of homozygous Brattleboro fetuses $(n=10)$

\begin{tabular}{lccc}
\hline & Vasotocin (AVT) & Vasopressin (AVP) & Oxytocin \\
\hline $\begin{array}{l}\text { Wistar } \\
\text { (day 21 of pregnancy) }\end{array}$ & $3.49 \pm 0.69$ & $9.27(0.51)$ & $0.42(0.04)$ \\
$\begin{array}{c}\text { Brattleboro } \\
\text { (day 22 of pregnancy) }\end{array}$ & $0.21 \pm 0.21$ & 0.00 & $0.78(0.08)$ \\
\hline
\end{tabular}

The peptides were determined in $0.1 \mathrm{~N} \mathrm{HCl}$ extracts of fetal pituitaries (for procedure see [9]) by specific and sensitive (up to $0.5 \mathrm{pg} /$ tube) radioimmunoassays based on [25]. For the AVT assay, anti-AVP No. 758 was used (cf. Table 1) after purification for 3 times by means of oxytocin containing agarose beads (cf. [19]), in order to remove the strong cross reaction with oxytocin. After purification the cross reaction with AVP or oxytocin were both less than $0.05 \%$. For the AVP assay, anti-AVP "E april" (cf. [25]) was used. The cross reactivity with AVT being less than $0.25 \%$ and with oxytocin less than $0.05 \%$. For the oxytocin assay O-2 D (cf. [19]) was used. The cross reaction with AVT was less than $0.05 \%$ and with AVP less than $0.005 \%$. Because pregnancy lasts 1 day longer in Brattleboro rats than in Wistars, this former litter was studied 1 day later. Note that neither AVT, nor AVP are present in the Brattleboros.

the cross reacting compound was not found in Brattleboro fetuses at term, being homozygous for diabetes insipidus, this could be the compound that was stimulating the course of labour.

Radioimmunoassay confirmed the presence of AVT in the fetal Wistar pituitary and its absence in the homozygous Brattleboro fetus at term. In addition, arginine-vasopressin (AVP) was only found in the Wistar fetuses, whereas the oxytocin content was lower in the Wistar than in the Brattleboro fetal pituitaries (Table 2). The small amount of AVP was apparently not sufficient to be demonstrated by immunofluorescence with a purified antibody, while oxytocin could even not be localized with the non-purified antibody. Pavel [23] concluded from the long lasting AVT production of cultured human fetal pituitaries, that AVT would be produced in glial elements. Immunofluorescence microscopy, using antivasopressin in Wistar fetuses at term, revealed the presence of the cross reacting compound, which is most probably AVT in the supraoptic and paraventricular nucleus and in the hypothalamo-neurohypophyseal tract. This argues for a neuronal source of AVT. This was confirmed by immunoelectronmicroscopical studies that showed the presence of the cross reacting compound in $140 \mathrm{~nm}$ neurosecretory vesicles in the fetal neuro- hypophysis with non-purified antivasopressin, while non-purified oxytocin revealed no staining whatsoever (Fig. 2). Thus, AVT seems to be produced by the fetal hypothalamo-neurohypophyseal system in the very same way as AVP and oxytocin in the adult. At what moment, and by what mechanism the switch from AVT to AVP or oxytocin production is made, is not yet known.

Because of the presence of AVT in fetal Wistar rats, the low, if any, pituitary presence in homozygous Brattleboro fetuses and the oxytocic activity of AVT on the rat uterus at term [7], this hormone may well be the fetal hormone that is involved in the acceleration of the course of labour. Experiments are currently in progress using antibodies raised specifically to AVT.

\section{DISCUSSION}

Recent findings stress the idea that important differences exist between fetal and adult neuroendocrinology. Two examples of hormones probably operating only in the fetal situation were given in the present paper, i.e. the presence of $\alpha-\mathrm{MSH}$ in the human fetal pituitary and of AVT in the fetal Wistar neurohypophysis. $\alpha-\mathrm{MSH}$ is thought to be absent in the pituitary of adult man except during pregnancy (see

Fig. 2. Electronmicrographics of a Wistar fetal neurohypophysis at term, i.e. day 21 of pregnancy. The pituitary was fixed in $2.5 \%$ glutaraldehyde and $1 \%$ paraformaldehyde in Sörensen phosphate buffer $(\mathrm{pH} 7.4)$ for $60 \mathrm{~min}$ and postfixed in $1 \% \mathrm{OsO}_{4}$ in Sörensen buffer for $90 \mathrm{~min}$.

(a) This section was counterstained by both, $2 \%$ uranylacetate in $50 \%$ ethanol for $30 \mathrm{~min}$ and by lead citrate for $5 \mathrm{~min}$. Although in this figure most fibres contain neurosecretory granules (mean diameter $140 \mathrm{~nm}$ ), the majority of fibres in the fetal pituitary are devoid of such granules. $\mathrm{c}=$ clear vesicles, $\mathrm{e}=$ extracellular space in which collagen fibres (co) can be observed; $\mathrm{mt}=$ microtubuli, $\mathrm{mch}=$ mitochondria, $\mathrm{n}=$ neurosecretory granules, $\mathrm{p}=$ processes of pituicytes in which lipid droplets [1] appear. For immunolocalization of the compound which was cross reacting with non-purified antivasopressin, ultrathin sections were incubated in $5 \% \mathrm{H}_{2} \mathrm{O}_{2}$ for $3 \mathrm{~min}$ and further treated according to the unlabeled antibody enzyme method [26].

(b) Incubation with antivasopressin (No. 126, dilution 1:400). Note the positive neurosecretory granules; $\mathrm{nu}=$ nucleus of a pituicyte.

(c) Incubation with anti-oxytocin (No. O-2 C, dilution 1:400). Note the negative neurosecretory granules (arrows). A similar negative picture was obtained with control plasma. The same results were obtained with all plasmas diluted 1:80. In this dilution O-2 C is strongly cross reacting with AVP in adult Wistars. Therefore the stained compound is most probably AVT. 


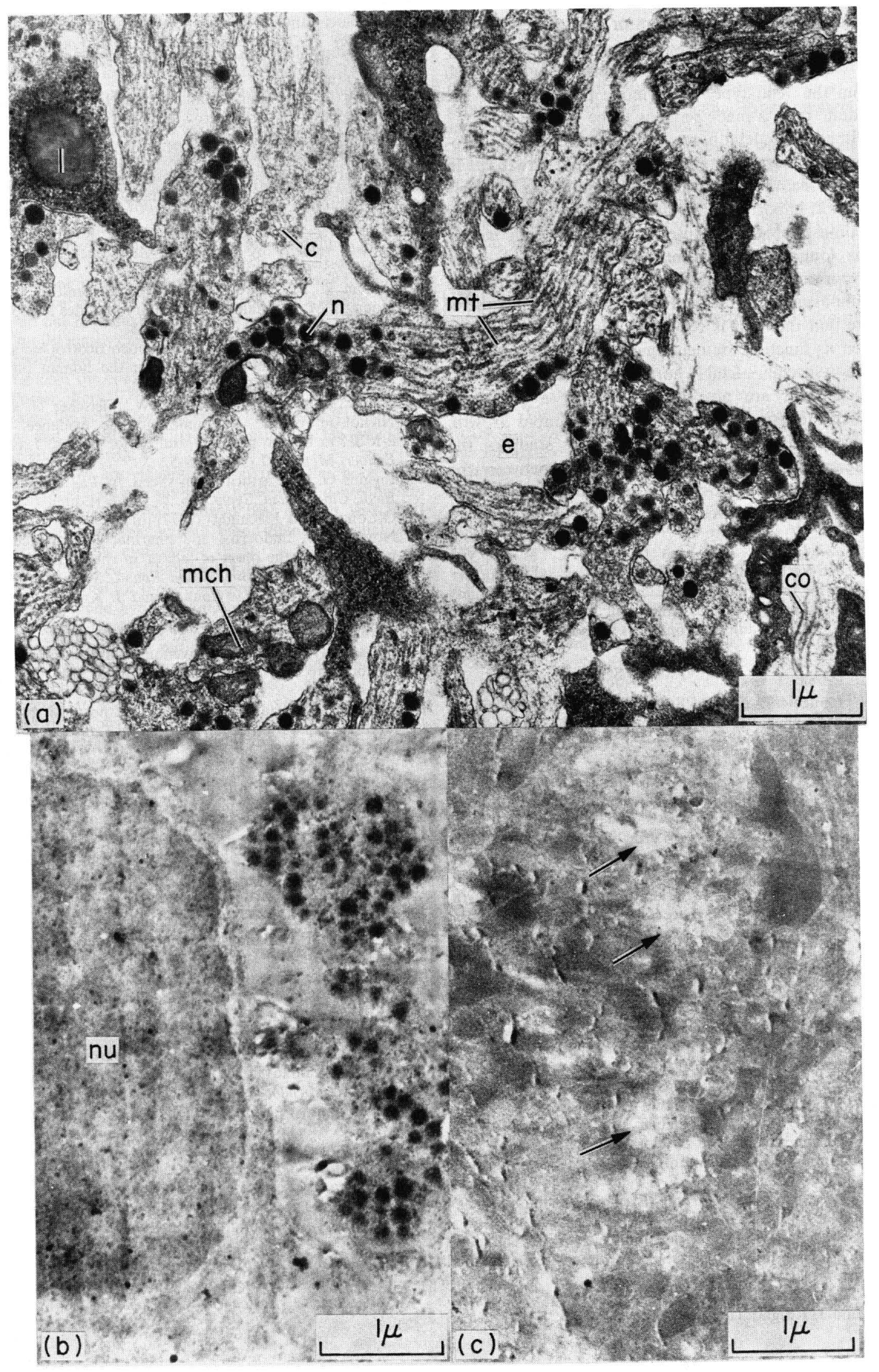


before). AVT, although present in the adult pineal gland, is thought to be absent or only present in very small quantities in the adult neurohypophysis [24]. At what moment and by what mechanism the switch from the 'fetal type' of hormone production to the "adult" type is made remains to be elucidated.

In addition, fetal hormones can be involved in processes that are different from the adult situation. In this respect the fetal brain and pituitary are probably also of importance. In human anencephalics and brain-aspirated rat fetuses a lower placenta weight was found $[1,3]$. Placenta weight increased again after injection of $\alpha-\mathrm{MSH}$ or growth hormone into the brain-aspirated rat fetuses [5]. It seems possible therefore that the fetus is regulating placenta weight, and even its function, by neuroendocrine mechanisms. In fact, specific fetal hormones and endocrine mechanisms are not illogical during a period in which the fetus is influencing its own rapid growth, differentiation and labour. A systemic study of the differences rather than the similarities between the adult and fetal endocrine systems might in view of the present data reveal many unexpected results.

Acknowledgements-We wish to thank C. M. F. van Rheenen-Verberg, B. Fisser, P. J. Wolters and J. van der Velden for their valuable assistance, and the Netherlands Organization for the Advancement of Pure Research, Z.W.O. for the additional financial support.

\section{REFERENCES}

1. Honnebier W. J. and Swaab D. F.: The influence of anencephaly upon intrauterine growth of fetus and placenta and upon gestation length. J. Obstet. Gynaec. Br. Commonw. 80 (1973) 577-588.

2. Honnebier W. J., Jöbsis A. C. and Swaab D. F.: The effect of hypophysial hormones and human chorionic gonadotrophin (HCG) on the anencephalic fetal adrenal cortex and on parturition in the human. $J$. Obstet. Gynaec. Br. Commonw. 81 (1974) 423-438.

3. Swaab D. F. and Honnebier W. J.: The influence of removal of the fetal rat brain upon intrauterine growth of the fetus and the placenta and on gestation length. J. Obstet. Gynaec. Br. Commonw. 80 (1973) 589-597.

4. Swaab D. F. and Visser M.: A function for $\alpha$-MSH in fetal development. Proceedings Symposium Control, Chemistry and Effects of MSH, Amsterdam, 1976. (1977) (in press).

5. Honnebier W. J. and Swaab D. F.: Influence of $\alpha$-melanocyte-stimulating hormone $(\alpha$-MSH), growth hormone $(\mathrm{GH})$ and fetal brain extracts on intrauterine growth of fetus and placenta in the rat. J. Obstet. Gynaec. Br. Commonw. 81 (1974) 439-447.

6. Swaab D. F. and Honnebier W. J.: The role of the fetal hypothalamus in development of the feto-placental unit and in parturition. In Integrative Hypothalamic Activity (Edited by D. F. Swaab and J. P. Schadé). Progress in Brain Research. Elsevier, Amsterdam, Vol. 41 (1974) pp. 255-280.

7. Swaab D. F., Boer K. and Honnebier W. J.: The fetal hypothalamus and pituitary in the onset and the course of parturition. In The Fetus and Birth, Ciba Found. Symp. No. 47, 1976 (Edited by M. O'Connor and J. Knight). (1977) (in press).
8. Kittinger G. W.: The endocrine regulation of fetal development and its reaction to parturition in the rhesus monkey. In The Fetus and Birth, Ciba Found. Symp. No. 47, 1976 (Edited by M. O'Connor and J. Knight). (1977) (in press).

9. Swaab D. F., Visser M. and Tilders F. J. H.: Stimulation of intrauterine growth in rat by $\alpha$-MSH. $J$. Endocr. 70 (1976) (in press).

10. Visser M. and Swaab C. F.: $\alpha-\mathrm{MSH}$ in the human pituitary. In Proceedings Symposium Control, Chemistry and Effects of MSH, Amsterdam, 1976. (1977) (in press).

11. Honnebier W. J. and Swaab D. F.: Regulation of growth velocity by the foetus. Acta Endocr. (Suppl.) 199 (1975) 97.

12. Silman R. E., Chard T., Lowry P. J., Smith I. and Young I. M.: Human foetal pituitary peptides and parturition. Nature 260 (1976) 716-718.

13. Scott A. P. and Lowry P. J.: Adrenocorticotrophic and melanocyte-stimulating peptides in the human pituitary. Biochem. J. 139 (1974) 593-602.

14. Abe K., Island D. P., Liddle G. W., Fleischer N. and Nicholson W. E.: Radioimmunologic evidence for $\alpha-\mathrm{MSH}$ in human pituitary and tumor tissues. J. clin. Endocr. Metab. 27 (1967) 46-52.

15. Liggins G. C.: Parturition in sheep. In The Fetus and Birth, Ciba Found. Symp. No. 47, 1976 (Edited by M. O'Connor and J. Knight) (1977) (in press).

16. Novy M. J.: Endocrine and pharmacological factors which modify the onset of labour in rhesus monkeys. In The Fetus and Birth, Ciba Found. Symp. No. 47, 1976 (Edited by M. O'Connor and J. Knight) (1977) (in press).

17. Nathanielsz P. W., Comline R. S. and Silver M.: Uterine activity following intravenous administration of oxytocin to the foetal sheep. Nature 243 (1973) 471-472.

18. Chard T.: The fetal hypothalamus and posterior pituitary in the initiation of labour. In The Fetus and Birth, Ciba Found. Symp. No. 47, 1976 (Edited by M. O'Connor and J. Knight) (1977) (in press).

19. Swaab D. F. and Pool C. W.: Specificity of oxytocin and vasopressin immunofluorescence. J. Endocr. 66 (1975) 263-272.

20. Swaab D. F., Pool C. W. and Nijveldt F.: Immunofluorescence of vasopressin and oxytocin in the rat hypothalamo-neurohypophyseal system. $J$. Neural Transm. 36 (1975) 195-215.

21. Ewy Z. and Rząsa J.: Effect of vasotocin and oxytocin on contractility of the oviduct and on blood pressure in the hen. Acta physiol. pol. 19 (1968) 407-416.

22. Skowsky R. and Fisher D. A.: Immunoreactive arginine vasopressin (AVP) and arginine vasotocin (AVT) in the fetal pituitary of man and sheep. Clin Res. 21 (1973) 205.

23. Pavel S.: Vasotocin biosynthesis by neurohypophysial cells from human fetuses. Evidence for its ependymal origin. Neuroendocrinology 19 (1975) 150-159.

24. Rosenbloom A. A. and Fisher D. A.: Radioimmunoassayable AVT and AVP in adult mammalian brain tissue: comparison of normal and Brattleboro rats. Neuroendocrinology 17 (1975) 354-361.

25. Dogterom J., Van Wimersma Greidanus Tj. B. and De Wied D.: Histamine as an extremely potent releaser of vasopressin in the rat. Experientia 32 (1976) 659-660.

26. Van Leeuwen F. W.: Immunolocalization of oxytocin and vasopressin at the light and electron microscopic level in rats. J. Histochem. Cytochem. 24 (1976) 618. 


\section{DISCUSSION}

Macnaughton. I just wonder how much stress you can place on your liver data in the anencephalic. The length of labour now is so artificial that one can never place any reliance on anything being natural as far as the length is concerned. I think yesterday you mentioned that this was over a period of 10 years you had collected these anencephalics and I would have thought there were so many changes in management that this would really make it impossible for you to say anything about the length of labour.

Swaab. Indeed, clinical data are always difficult to interpret, even if you match the controls as well as possible. This is one of the reasons that we always try to confirm the clinical findings in our rat experimental model, where we do not have such problems. The data obtained in the rat model match the clinical findings very nicely, until now. Therefore we think it is appropriate to draw the conclusions from the clinical material as we did.

Posner. Very interesting paper. Do you know of any data on the actual measurement of $\alpha \mathrm{MSH}$ in fetal circulation, and in particular its relationship to the period of accelerated growth.

$S w a b b$. We are elaborating that particular point now. The only thing I can say up to now is that a preliminary study, using a bioassay, showed that there is less MSH activity in anencephalic cord blood than in normal controls (Honnebier and Swaab, 1975).

Jaffe. I noticed in that very extensive list of substances which you tried for the growth promoting effects that you had used crude hypothalamic extract. Did you also use just a crude total pituitary extract. I did not notice that anywhere. I only saw purified preparations.

Swaab. No, we did not, because the pituitary hormones were available in a purified or synthetic form.

Friesen. It was difficult for us to see the data on the effects of various preparations of MSH. I wonder if you could just tell us the weight gain that you observed with the injections and how much MSH was injected.

Swaab. We injected $\alpha$-MSH in physiological saline subcutaneously directly into the fetus in doses of either 15 or 150 micrograms (Honnebier and Swaab, 1974). The weight gain obtained was as a mean about $50 \%$ of the difference between brain-aspirated and sham operated animals (Swaab and Honnebier, 1973). So we could not restore the entire difference in body weight between brainless fetuses and intact fetuses. However, we do not know yet whether this is caused by the fact that we are only injecting the fetuses once and weighing them two days later. The peptide is not given as a long acting depot preparation so the effect might last only shortly.

Friesen. How does that dose compare with the content of MSH in the pituitary at that time? Is it 10 times greater?

Swaab. The fetal pituitary content as measured by bioassay is maximal $25 \mathrm{ng}$ /pituitary (Swaab et al., 1976), so 600-6000 times less than the dose given. But the pituitary content does not give us any indication about the production rate in the fetus, while at the other hand we do not know how quickly the injected dose of $\alpha$-MSH disappears.

Friesen. Along the same lines, what was the neutralizing capacity of the antibody that was injected; would it neutralize all of the MSH present?

Swaab. How would you suggest to measure that?

Friesen. Like competitive displacement studies in radioimmunoassay type set up.

Swaab. The amount of labeled $\alpha-\mathrm{MSH}$ that would bind to the dose of antibody given to the fetuses will be in the nanogram range under radioimmunoassay circumstances. In vitro measurement of that kind do, however, not tell us anything about the in vivo situation. What we would like to know is how much the given dose of antibody neutralizes the endogenously produced $\mathrm{MSH}$ in vivo and that is not known.

Grumbach. In addition to your evidence in regard to $\alpha$-MSH, the only hormone of which there is indirect evidence that it may effect fetal growth is insulin, clearly in both the human anencephalic fetus and in your experimental rat model removal of the brain hypothalamus could effect insulin secretion. Have you looked at insulin in your rat model. As you know Dr. Piquant and Professor Jost have been able to by injection of insulin into the rat fetus to increase their weight.

Swaab. We have injected insulin directly into brainaspirated and intact rat fetuses, but we could not get any increase in weight (Swaab and Honnebier, 1974). In addition, decapitation of rabbit fetuses increases fetal plasma insulin levels, and glucose induces an increased insulin release from the pancreas of these animals (Jack and Milner, 1972). So it does not seem as if the stimulating action of the fetal brain and pituitary on intra-uterine growth is mediated through insulin. 